\title{
(More) Lessons Learnt from the Deployment of Production Worldwide Grid Services for the Large Hadron Collider at CERN
}

\author{
J.D. Shiers, CERN, Geneva, Switzerland
}

\begin{abstract}
This paper describes the main lessons learned in deploying production services for the Large Hadron Collider (LHC) at CERN. We cover the principle difficulties faced in bringing this multi-Grid service to full production status and how they were resolved. We also list remaining challenges, how they will be addressed, as well as possible future directions for long-term sustainable multi-disciplinary einfrastructures. Finally, we introduce a simple classification of applications that use -or could use - Grid infrastructures and examine how they could coexist and the inherent mutual benefits.
\end{abstract}

\section{INTRODUCTION}

In order to process and analyze the data from the world's largest scientific machine, a worldwide grid service - the Worldwide LHC Computing Grid (LCG) [1] - has been established, building on two main production infrastructures: those of the Open Science Grid (OSG) [2] in the Americas, and the Enabling Grids for E-sciencE (EGEE) [3] Grid in Europe and elsewhere.

The machine itself - the Large Hadron Collider (LHC) - is situated some $100 \mathrm{~m}$ underground beneath the French-Swiss border near Geneva, Switzerland and supports four major collaborations and their associated detectors: ATLAS, CMS, ALICE and LHCb.

Even after several levels of reduction, some 15PB of data will be produced per year at rates to persistent storage of up to $1.5 \mathrm{~GB} / \mathrm{s}$ - the LHC itself having an expected operating lifetime of some $10-15$ years. These data will be analyzed by scientists at close to two hundred and fifty institutes worldwide, using the distributed services that form the Worldwide LHC Computing Grid (WLCG).

Over the past 3 years, the WLCG services have undergone a series of so-called 'service challenges' designed to ramp the service up to the required degree of availability and performance and to trigger medium / long term planning of the various sites and regions involved.

An important component of these challenges has been the strong participation of the experiments themselves in progressively and systematically testing the various components of the services, adapting their frameworks to them and building up to full end-to-end tests - the so-called 'Full Dress Rehearsals' - scheduled to take place starting in the

Manuscript received November 4, 2007.

J. D. Shiers is with the European Organisation for Nuclear Research (CERN) (e-mail: Jamie.Shiers@ cern.ch). summer of 2007. We discuss the close collaboration between the service providers and the experiments and highlight techniques that have been especially effective in resolving service problems - which in such a complex, distributed system can often be extremely hard to resolve.

Detailed service and availability targets have been drawn up by the WLCG collaboration and form part of the Memorandum of Understanding (MoU) that is signed by all participating sites. The level of service delivered is regularly reviewed by the management bodies of the project.

We summarize below the main lessons learnt from deploying WLCG production services. In particular, we discuss how the somewhat ambitious targets laid out in the MoU are addressed and the various deployment strategies that are required. A strong focus is placed on Reliability, Scalability and Accountability, which together lead to both manageability and usability. Techniques for zero user-visible downtime for the main types of service intervention are described, together with pathological cases that need special treatment. The requirements in terms of scalability are analyzed, calling for as much robustness and automation in the service as possible. The different aspects of accountability - which covers measuring / tracking / logging / monitoring what is going on and has gone on - are examined, with the goal of attaining a manageable service.

The current status of the services is described, together with the outlook for the future, not only for the WLCG itself, but for large-scale grids in general, particularly in the context of moving to sustainable and scalable long-term infrastructures. Finally, a simple analogy is drawn with the Web in terms of usability - what do we need to achieve to cross the chasm from small-scale adoption to ubiquity?

\section{BUILDING THE SERVICE - A MOVING TARGET}

Over the past three years, the computing service required by the LHC experiments at CERN has been deployed across some 200 sites in many countries around the world. The experience of this deployment and concurrent hardening is described in detail in [4]. The computing needs of the LHC experiments and the manner in which these are addressed by a worldwide Grid are explained in [5]. To summarize the main difficulties:

- During this period, the computing models of the experiments were undergoing constant development and validation. Experience with the service that was trying to address the requirements of these models often resulted in changes, which in turn meant revised requirements that had to be taken into account by the 
middleware and often in terms of service deployment. Some examples of major changes that had to be accounted for include the deployment model of the file catalog as well as the data models of all of the experiments, which underwent significant evolution during this deployment period and had a corresponding impact on the architecture of the reliable File Transfer Service;

- Simultaneously, the production Grid infrastructure was being built up - hardware was being deployed, procedures developed, communication channels setup and improved - in other words, the entire operations infrastructure was being designed and built.

Thus we were simultaneously trying to enter more than one "brave new world" at the same time. Whilst this has been an extremely hectic period, the startup schedule of the LHC gave a clear target by which production services needed to be ready (well in advance of the actual startup for essential preparation and validation of the experiments' production systems.) Although commissioning activities of the accelerator itself have uncovered a number of problems that are rapidly being addressed, the computing service needs to be fully established well in advance of first data - to permit the commissioning of the detector and offline systems, which clearly cannot be left to the last minute.

\section{THE TARGETS FOR THE SERVICE}

In terms of the WLCG, the services that a given site must offer, the resources that should be made availability to given virtual organizations (VOs), as well as the service availability and maximum time in responding to problems are detailed in the WLCG Memorandum of Understanding (MoU) [6] that is signed by each institute that is a member of the collaboration. The status of the services is monitored on an hourly basis by an automated framework with trouble tickets raised in case of problems. The availability of the individual services at a site together with the average availability on a monthly basis is reviewed by the management board of the project, with sites that do not meet their targets required to provide a detailed explanation for the problems seen as well as the steps that are being taken to resolve them, These measures have resulted in a steady improvement in the basic services and the tests are complemented by $\mathrm{VO}$-specific ones and a flexible monitoring framework has been established - design to complement, rather than replace - existing $\mathrm{VO}$ and site monitoring. The target service levels and time to respond are somewhat aggressive - up to $99 \%$ availability is demanded of the accelerator centre at CERN and this of complex, compound storage, computational and networking services.

Notwithstanding the sometimes tumultuous changes that have taken place during the deployment and ramp-up phase of the project, we have nevertheless arrived at a few well-tested and straightforward strategies for delivering services of the needed reliability, although this has typically had to be retro-fitted to existing services. We suggest that this development, deployment and operational experience is sufficiently generic to be of value to future Grids and indeed similar or at least equivalent strategies will be required if the appropriate service level is to be offered with an affordable level of manpower.

\section{RUNNING ROBUST AND RELIABLE SERVICES}

Starting in August 2005, and based on the service levels defined in the WLCG MoU, an a priori analysis of the Tier0 WLCG services was performed. This analysis was performed as part of the commissioning of the WLCG service, driven to a large degree by a series of four "service challenges", aimed at "achieving the goal of a production quality world-wide Grid that meets the requirements of the LHC experiments in terms of functionality and scale." The above-mentioned analysis targeted not only the hardware needs, but also the middleware requirements, operational procedures and all other service aspects involved in setting up robust and reliable services. In addition, the feedback and experience from the early months of Service Challenge 3 - the first that included an attempt to provide a full set of services required by the LHC experiments as well as explicit experiment testing - called for a significant number of service updates. In order to perform these, a "long shutdown" of several days was scheduled during October 2005. It was well understood that such intervention could not normally be performed on a production service, but this was felt to be the least intrusive method available at that time to perform the numerous pending upgrades - including not only deployment of new middleware releases, but also network reconfiguration, hardware moves and reallocation. Unfortunately, sufficient hardware was still unavailable to redeploy the services in an optimal manner, and their redeployment continued over a period of many months. This was first done using a regular "intervention slot" - simplifying not only scheduling of such interventions with the experiments but also their production planning. However, it was soon realized that the coupling between the various services - not to mention their impact that in many cases extended way beyond the host site and was often Grid-wide - called for a less intrusive manner of performing such changes.

More recently, a re-analysis - an "a posteriori analysis" - was performed to take into account not only the prolonged experience with the production service, but also progress in making at least the most common interventions transparent to users. This analysis is still on-going and is likely to be extended to experiment-specific services that require the same degree of reliability of those built on Grid middleware components, but already consensus on the methodologies that have proved effective for delivering robust services has emerged. Before these are detailed, we contrast Grid to cluster / mainframe services.

\section{THE WHOLE IS GREATER THAN THE SUM OF THE PARTS}

The (W)LCG Technical Design Report (TDR) [7] lists two motivations for adopting a Grid solution. These are as follows:

1. Significant costs of [ providing ] maintaining and upgrading the necessary resources ... more easily handled in a distributed environment, where individual institutes and ... organisations can fund 
local resources ... whilst contributing to the global goal

2. ... no single points of failure. Multiple copies of the data, automatic reassigning of tasks to resources... facilitates access to data for all scientists independent of location. ... round the clock monitoring and support.

For funding reasons, the first argument is clearly extremely important - for the reason stated in addition to the fact that many of the institutes involved are multi-disciplinary. Thus, not only for resource sharing within a site but also to bolster the scientific and intellectual environment in the collaborating countries, such a scenario is much healthier than one where all resources are concentrated at the host laboratory (and acquired locally).

The second argument needs further analysis and is indeed similar to the $3^{\text {rd }}$ criterion in Ian Foster's checklist [8]:

“... to deliver nontrivial qualities of service. (A Grid allows its constituent resources to be used in a coordinated fashion to deliver various qualities of service, relating for example to response time, throughput, availability, and security, and/or co-allocation of multiple resource types to meet complex user demands, so that the utility of the combined system is significantly greater than that of the sum of its parts.)"

With the exception of services and processing that is performed at the Tier0 site, the fact that much of the data - e.g. with the exception of Monte Carlo data in a given Tier2's output buffer - is replicated at several or many sites, the partial or even total failure of a site should not stop the associated production or analysis. Similarly, some of the services - such as the FTS - are already designed to cater for service interruptions at source and/or sink site. For example, if the storage element (SE) at a given site is about to enter scheduled maintenance, the corresponding FTS channels that source or sink data in that SE can be paused. This still allows new transfer requests to be queued, but they will not be attempted until the channel is re-opened, avoiding wasting bandwidth on transfers that are bound to fail and potentially reducing the background load on support staff (analysing "fake" failures.)

\section{BUILDING ROBUST SERVICES}

Robust services can only be delivered through careful planning complemented by a combination of techniques, including the appropriate steps at application design and implementation level, as well as at the deployment and operational stage. We describe below very simple techniques that have proven extremely effective and widely applicable in designing and delivering reliable services with a reasonable level of effort and - importantly - largely avoiding fire-fighting and panic.

Two mindsets that are particularly important in this respect are:

- Think service - a service is far more than a middleware release in a 'production' repository;
- Think Grid - a Grid is the ultimate distributed computing system (so far). A change to a service deployed at a given site or site(s) may well have an impact far wider than the local community and must be planned and announced accordingly.

Before we list the techniques that are in daily use for deploying and operating the WLCG service, we consider some of the issues related to failures and support calls, together with their associated costs.

Consider, for example, the reliable File Transfer Service. Given the expected data volumes and rates, a typical LHC experiment would transfer some $10^{5}-10^{6} 1 \mathrm{~GB}$ files per day many more if analysis data and calibration datasets were also included. The percentage of such transfers that fail in such a way that human intervention is required must be extremely low, particularly as the problems seen after automatic retries are often complex and time consuming to resolve. Other examples come from user support costs. A ticket that a ticket processing manager spends 1 hour on (and many take much more to solve) has a real and non-negligible cost associated with it. Not all such problems can be avoided purely through good documentation and robust services, but there is clearly very strong motivation to do so. Finally, any operational issues that require human follow up must be reduced to the absolute minimum - anything that can be documented in English (or indeed any other language) can also be programmed as a script or in a higher level language - computers are simply much better at doing repetitive tasks rapidly than humans, whose particular analytical skills are best used elsewhere.

\section{CHECK-LIST FOR NEW SERVICES}

Before a new service is deployed - be it in a Grid or non-Grid environment - a straightforward checklist has been established that has proven invaluable in ensuring that the resultant services are of the required quality. Ideally, this work starts well prior to deployment - the middleware must be designed and written with reliability in mind. This includes details such as error messages and logging - this must be consistent and in an agreed place to which the necessary support teams have access if required (the latter is non-trivial in the case of crosssite services). The application must be designed to cope with "glitches" - e.g. short-lived problems with services on which they depend and which are simply unavoidable in a distributed environment. Where possible, the ability to share the load across multiple load balanced servers offers numerous advantages, including transparency to many common service interventions and even middleware upgrades. In the case of a database backend, the ability to re-establish a connection and assuming a database cluster - failover transparently from one node to another are highly desirable, if not mandatory, features. The appropriate hardware must obviously be allocated - avoiding (except in cases such as batch worker nodes) single points of failure through power supplies or feeds, network connections and so forth. Finally, a minimum set of operational procedures - including contact names and addresses - together with a basic set of tests (no contact, high 
load etc.) is needed. The necessary workflows also needed to be established in the support lines, together with diagnostic tests for the various levels of support / operations teams. Starting with these essentials, the service manager can readily add more tests and procedures as experience shows are required.

\section{DAILY AND WeEKLy OPERATIONS MEETINGS}

One of the key secrets to running smooth services is a regular operations meeting. This has been in place at CERN since decades before the Grid and use to be performed by vendor (CERN having a number of large mainframes / clusters at that time). In recent years, these meetings have been extended to cover the Grid world, with a clear impact on the state of the Grid services. On occasion, people have expressed 'disappointment' that there is not an atmosphere of mad panic / firefighting at these meetings - but this is precisely the point these meetings are to ensure a smooth service, exactly the opposite of firefighting. Instead of being an overhead, these meetings act as an excellent point of information exchange, and in fact significantly reduce the amount of time spent on identifying and debugging problems. The meetings typically take around 10 minutes - slightly longer on Mondays - and quickly run through alarms and problems seen since the last meeting. If not immediately solved, the problems are assigned to a system administrator or technical expert as appropriate. More importantly, they allow weaknesses in the services such as lack of adequate monitoring or alarms - and peer pressure proves a very effective mechanism for ensuring that these holes are rapidly plugged. Once a week, any outstanding tickets against the CERN Regional Operations Centre are reviewed, again ensuring that problems are not left unaddressed for prolonged periods. Another important topic that is reviewed daily is any interventions scheduled for that day, or any foreseen in the coming days. It cannot be stressed too highly how important adequate preparation for interventions has repeatedly been proved to be - it is not just a question of informing fellow service providers and users, but also ensuring that the intervention proceeds smoothly. All to often, a well debugged procedure runs into problems (often because it is not strictly followed, or as the availability of needed colleagues for a given step has not been checked), turning a smooth or even "transparent" intervention into a prolonged downtime that may even need a further intervention to adequately complete. In the worst cases, unannounced "transparent" interventions have resulted in severe service degradation that have led to extreme user dissatisfaction and have been extremely costly in terms of manpower to resolve. We have therefore agreed simple procedures for announcing scheduled interventions of various lengths, as well as unscheduled interventions. Equally importantly, an announcement through the agreed channels is required when the service is fully restored (or periodic announcements in case of prolonged problems), as well as an open post-mortem, recording any unforeseen problems, their resolutions and lessons for the future.
These daily - primarily site-oriented (see caveat above) meetings are complemented by weekly joint operations meetings with all the main sites that have a similar agenda but also include VO-specific issues. Finally, less frequent meetings are held to ensure that the operations tools adequate address the needs of the community. These meetings are typically held bi-annually.

\section{Key TeChNiques}

The main techniques that are used in conjunction with the standard operations procedures are both simple and wellunderstood:

- Understanding the impact of downtime or degradation to service. In some cases, it may even be acceptable for a problem only to be resolved the next working day whereas in others this would clearly be unacceptable: resources being limited, the effort (and money) needs to be focused in the right places;

- The use of database clusters for middleware components that have persistent state (together with the appropriate deployment and application development strategies);

- Load-balanced servers for the middle tier.

These techniques not only allow services to be resilient to single (or even multiple) component failure, but permit many of the common interventions to be performed with zero uservisible downtime. These include operating system, database or middleware upgrade or security patches as well as the addition of new hardware / replacement of old or failure nodes. In the case of the best behaving applications, these techniques have been fully supported for a number of years. Further work is required to make all of the main services sufficiently resilient this is currently underway, being driven by the priorities of the experiments.

\section{Critical Services}

The services that an LHC experiment relies on to run its production include a number of important VO-specific components over and above the standard Grid middlewarebased ones. If one of these services is down or impaired, the experiment is impacted at least as severely as if one of the key Grid services was down. It is therefore essential to address the reliability of these components. A proposal that has yet to be put in place is to treat these services in the same manner as the standard Grid services, including techniques for writing robust services as well as their deployment and operation. Particularly in the early months and years of data taking, it is likely that there will still be some residual instability in some of these services and it is proposed that in the key areas of storage, Grid data management and databases for physics that an oncall service is established. This would allow technical specialists to be contacted $24 \times 7$ in case of problems that cannot be resolved using the standard documented procedures. It will clearly provide significant motivation to further improve the robustness of the services and it is foreseen that the need for such on-call be reviewed annually. For other important services, for which a permanent on-call rota is not justified, named contacts within each of the experiments will be able to 
call out an expert by passing through the console operators. Such interventions need to be relatively rare and will also be regularly monitored to understand both the need and sustainability of the system.

\section{MONitoring, LOGGING AND REPORTING}

These related but distinct aspects of running a service are often confused. Furthermore, the specific information that should be presented depends very much on the target audience. A common trap is to try and build everything into a single tool, which continues to grow until it is un-maintainable and even unusable. For example, a funding agency may be concerned with how well the resources provided being used. A VO manager may wish to see how well their production is proceeding. A site administrator on the other hand may simply want to see if his or her services up and running and meeting the agreed MoU targets. The on-duty operations team will typically want to know if there any outstanding alarms. Finally, an LHCC referee may want to see how the overall preparation progressing with any areas of concern highlighted.

Nevertheless, much of the information that would need to be collected is common and so it is important to separate the collection from presentation (views...), as well as the discussion on metrics. It is precisely this approach that is being adopted - with some success - by the LCG monitoring working groups that were created one year ago. In addition, the issue of improved and consistent logging is being actively pursued by the middleware developers - the status of both of these issues being presented at the EGEE'07 conference in Budapest [9][10][11].

\section{MIND THE GAP}

During the above conference, a number of service problems came up that highlighted the need for well documented - and followed - procedures, as well as excellent communication. To be explicit, three significant service problems came up in a single week - all of which were easily avoidable. These were as follows:

- A bug in Oracle client libraries - both documented and already fixed in production releases - caused a number of daemons to go into an infinite loop (after 248 days of uptime - the maximum number of clock-ticks (1/10s) that can be represented in a 32 bit integer). An analysis of the problem revealed that not only are there numerous methods for deploying Oracle client releases but also there was no consistent agreement for which of these to use, nor for moving to new versions;

- A change to the service availability algorithm - improved in principle at the various management boards - was released in production without being scheduled or even announced via the regular operations meetings. This caused significant knock-on effects in other service monitoring tools;

- A database house-keeping exercise resulted in an index being de-selected, with following service overload and meltdown.
Whilst it is unlikely that all such problems can be avoided in the future, we cannot afford to tolerate such a high rate of completely avoidable issues. Hopefully, the experience from these events will reinforce the widespread adoption of the simple and lightweight procedures that have been shown to work in exactly these situations.

\section{CAVEAT EMPTOR}

A final piece of cautionary advice concerns coupling between services and the sometimes unexpected consequences. Two concrete examples in this area relate to the choice of database synchronization technology that we have deployed. This is used for the file catalog middleware component and for detector and calibration alignment information - in both cases the corresponding information is kept in sync between the main sites with minimal delays. However, this has meant on one occasion that a key database feature had to be disabled with significant consequences on the ability to recover the service in case of accidental loss of data - and on another led to silent data corruption. On balance, the benefits certainly outweigh the drawbacks, but underline the need for openness and transparency - the reasons for choosing a specific release and the consequences must be clear to all, particularly in the case of complex, layered services.

\section{Conclusions on Robust SERvices}

Taken together, these techniques and procedures have been demonstrated to be sufficient to offer robust and resilient services, but are unfortunately often overlooked. We know how to run reliable services - this is not to say that no user support issues remain! The issue of support for large and diverse user communities of a system with the complexity of the Grid is certainly one of the challenges that will need to be addressed by future e-infrastructures. In particular, it is essential that we neither design nor use Grids in such a way that the unavailability of a single service renders a site - or worse the entire Grid - down. Such problems should, in the worst case, result in a small inefficiency of the overall Grid resources, rather than a downtime.

\section{REMAINING CHALLENGES FOR WLCG}

Despite many years of preparation - and indeed considerable and measurable progress - much remains to be done to the WLCG service before the LHC is fully operational. It should be stressed that the production service has been open since one year (and in fact in use for several years prior to that) and is used around the clock on a daily basis. However, a significant ramp-up in deployed resources is required - a factor of approximately five in processing and storage capacity for all participating sites, as well as an improvement of up to a factor of three in data export throughput. In addition, full production processing at 2008 scale for all 4 experiments must be supported.

As a reminder, the main responsibilities of the different tiers of the WLCG computing model are as follows: 
- Tier0 (CERN): safe keeping of RAW data (first copy); first pass reconstruction, distribution of RAW data and reconstruction output (Event Summary Data or ESD) to

Tier1; reprocessing of data during LHC down-times; - Tier1: safe keeping of a proportional share of RAW and reconstructed data; large scale reprocessing and safe keeping of corresponding output; distribution of data products to Tier2s and safe keeping of a share of simulated data produced at these Tier2s;

- Tier2: Handling analysis requirements and proportional share of simulated event production and reconstruction.

Whilst all experiments have carried out detailed testing, this has been largely performed on an experiment by experiment basis and not as a coordinated effort from online to offline for all VOs concurrently. It is agreed that the 2008 resources are made available for production use by April $1^{\text {st }}$ - given the ramp-up of up to 5 in scale, this is a significant challenge in its own right and unforeseen 'surprises' due to this increase in scale should not be ruled out.

The schedule for the LHC in 2008 is for first (single) beam in May 2008 with collisions in the July timeframe. However, the machine may not run with full operational stability in the initial months and one possible scenario is that the aggregate data rate for at least part of the year will be dictated by that coming from the interaction of cosmic rays with the detectors with peaks corresponding to the nominal rates from protonproton collisions. Both will depend on the degree to which zero suppression is possible - the upper limit being given by the capacity of the online systems. Aside from the technical challenges to be solved, there will be significant difficulties in agreeing schedules across so many sites and communities. This is being coordinated by a so-called Common Computing Readiness Challenge (CCRC), foreseen to include two formal test periods (February and May 2008), building on the commissioning activities already in progress in the individual experiments.

\section{WHO NEEDS A GRID?}

Broadly speaking, applications that need to use a Grid can be categorized as follows:

- Provisioned: the application needs significant quasidedicated resources for long periods (years, if not decades). Examples of this category include High Energy Physics experiments, astrophysics / astronomy etc;

- Scheduled: large amounts of resources are required for periods much shorter than the above - perhaps days or weeks. However, given the required scale, dedicated provisioning for such short periods is excluded. Examples in this category range from time-critical such as disaster response - to non-critical, that can be scheduled well in advance;

- Opportunistic: the least demanding area which can effectively soak up any availability resources. Much less time critical that the two previous areas.
Given the ostensibly differing needs of these application areas, can a single shared Grid infrastructure meet their requirements? What are the pros and cons of such an approach - in particular, what is the motivation for funding bodies to invest in such a shared infrastructure and why would application communities be willing to use it in this nonexclusive way?

Perhaps the most persuasive argument comes from the second category - both those that are time-critical and those that are not. Consider, for example, a disaster response scenario. By definition, one cannot know when such an event will take place, even if there might be times of increased risks for certain types of disaster. However, when such an event occurs, time is of the essence and any delay will have a corresponding effect in the response to any such problem. Governments and other organizations involved in disaster response cannot realistically be expected to have the necessary computing infrastructure on hot-standby in case of these hopefully rare events (in contrast to regularly used response teams, such as fire brigades and ambulances). Furthermore, unless the needed infrastructure is regularly exercised, it is likely that it will not be in a usable state on the hopefully rare occasion that it is needed. Further arguments in terms of economy can be found both in the areas of scheduled (non-time-critical) and opportunistic use. Finally, "provisioned" applications offer an excellent long-term load that can be guaranteed to ensure that the Grid infrastructure is permanently in full production status and evolves continuously to remain state-of-the-art and hence competitive.

XVII. GENERIC MIDDLEWARE VERSUS AN APPLICATION ORIENTED APPROACH

Experience shown in offering production services to a variety of applications shows that there is a clear distinction between "generic middleware" and those middleware components which require detailed knowledge of the computing model of the application area. Even within the WLCG community, the four LHC experiments have significant differences in their computing models which strongly impact on the suitability and usage of existing middleware components. As an example, only two (ATLAS and LHCb) explicitly use a generic file catalog component, but in very different ways (ATLAS as a 'local' file catalog, covering a Tier1-Tier2 cloud (in their nomenclature); $\mathrm{LHCb}$ as a global catalog with a $\mathrm{R} / \mathrm{O}$ replica (eventually) at all of their Tier1 sites (R/W master at the Tier0 - CERN. Another example is the gLite File Transfer Service, where again the computing models, whilst globally similar at the high level, differ sufficiently in detail that specific developments and deployment models had to be foreseen. Thus, we propose two categories of middleware: generic middleware, which is neutral to the computing models of at least several applications, and application-oriented middleware, which takes the latter into account. This means that the corresponding support must be organized on the same lines and that for successful operation of an application in the Grid environment first detailed analysis of the computing model(s) involved, followed by on-going application-oriented support, must be foreseen. This is a non-negligible cost that 
must be understood for a generic infrastructure, where Grid experts must work hand-in-hand with application experts. Any simplification or streamlining that can be performed in this area can be expected to have major benefits and should be considered a requirement if the Grid is reach the same level of ubiquity as the Web - even in its state as it was ten plus years ago.

\section{PROVISIONED APPLICATIONS}

The obvious (in this paper at least) - but far from only example of a provisioned application is that of a High Energy Physics experiment. As stated above, sites that are members of the WLCG collaboration are required to commit resources per VO that is supported by that site with a floating horizon of several years and the delivery of the pledged resources is followed by the LHC Computing Resource Review Board [12]. However, accounting data shows that these allocations are not strictly adhered to - other LHC VOs and non-HEP VOs are also able to make use of these resources during certain periods. However, HEP is by no means the only example of provisioned applications - others in this category include Astronomy / Astrophysics and Biomed.

\section{Scheduled APPLiCATIONS}

An example of scheduled applications include the periodic validation of releases of the GEANT4 [13] detector simulation package. This software system is widely used in the HEP community and two production releases are performed per year. The regression testing that is needed for these releases requires a significant amount of resources - of the order of 4 years of CPU time. This makes it a very natural candidate for using a production Grid, where resources are only needed for relatively short periods that can be planned well in advance. Another such example is the ITU spectrum allocation production that was performed as part of the ITU's Regional Radiocommunication Conference held in Geneva in 2006 [14]. The goal in this case was to perform the data processing required to allocate frequency according to different models during the conference itself - an activity that required some 400 PCs working in parallel. Both of these two applications can be considered non-time critical, in that the necessary productions can also be prepared well in advance - a significant simplification with respect to "disaster response" applications, where one cannot wait for the target application to be ported to the Grid, nor for the currently lengthy creation of an new VO (one does not know a priori what sort of disaster will strike, nor indeed where or when). Clearly, should a problem of this nature occur as rapid a response as possible is required and resources funded primarily for other less critical applications should be diverted with priority as required.

\section{Opportunistic GRID USAGE}

The numerous applications in this domain include flood prediction, atmosphere simulation and other Earth Science areas [15], as well as drug discovery and other clearly important areas, many of which are described in the EGEE II news area [16]. Whilst by no means the only examples, they can all use (very) large amounts of resources and are also all clearly important to fund. A number of different techniques exist to provide opportunistic access to resources - this includes "SETI@HOME" [17] style applications (which include LHC@HOME [18], that has played an important contribution to modeling of the LHC machine and selection of various parameters) - as well as other more "Grid-like" (according to Ian Foster's definition [8]) solutions. A number of different approaches to the opportunistic use of Grid resources together with associated application domains were shown during the recent EGEE '07 conference in Budapest.

\section{REMAINING CHALLENGES FOR GRIDS}

Grids have proven extremely effective for aggregating significant resources across many different management domains, offering unparalleled computing power in a relatively consistent manner. There is no doubt some entry threshold below which it does not reasonably make sense for an individual site to join a Grid and correspondingly economies of scale for larger sites. Thus, rather than fund a larger number of single disciplinary sites, there is motivation for a smaller number of centers of excellence - provided that the challenges of providing robust and usable services, together with an acceptably light-weight migration process to the Grid can be solved. Finally, the need for both generic middleware as well as application specific support needs to be emphasized. The former will hopefully reduce to something of the order of operating system support that is required today. On the other hand, application support can be expected to have strong benefits for many years to come and should be fully supported in future projects.

\section{CONCLUSIONS}

After many years of research and development followed by production deployment and usage by many VOs, worldwide Grids are a reality. There is significant interest in longer-term sustainable infrastructures that are possible with the current funding models and work on the definition of the functions of and funding for such systems are now underway. Using a very simple classification of Grid applications, we have briefly explored how the corresponding communities could share common infrastructures to their mutual benefit. Finally, the operational and support costs of Grids need to be contained and the difficulties in supporting new communities and their applications minimized. These and other issues are being considered by a design study for a long term e-infrastructure [19].

\section{ACKNOWLEDGMENT}

Many people have contributed to and continue to contribute to the ramp-up and delivery of WLCG Services. It is impossible - for simple space reasons - to mention every individual involved in this work. With apologies to those not explicitly mentioned, which includes regular participants in the LCG Service Coordination Meetings and the joint operations 
meetings - whilst gratefully acknowledging their work - the following warrant an explicit mention. The WLCG Service Coordination team consists of (in addition to the author): James Casey, Flavia Donno, Maarten Litmaath and Harry Renshall. Patricia Méndez Lorenzo coordinated and presented the "a posteriori" analysis of WLCG Tier0 services and Tim Bell coordinated the original "a priori" work. Ulrich Schwickerath, Miguel Coelho Dos Santos, Jean-Philippe Baud, Harry Renshall, Gavin McCance, Maria Girone and Dirk Düllmann all provided input to this recent analysis.

\section{REFERENCES}

[1] The Worldwide LHC Computing Grid (WLCG), http://lcg.web.cern.ch/LCG/.

[2] The Open Science Grid, http://www.opensciencegrid.org/.

[3] The Enabling Grids for E-sciencE (EGEE) project, http://public.euegee.org/.

[4] J. D. Shiers, "Lessons Learnt from Production WLCG Deployment", to appear in the proceedings of the International Conference on Computing in High Energy Physics, Victoria, BC, September 2007.

[5] The Worldwide LHC Computing Grid (worldwide LCG), Computer Physics Communications 177 (2007) 219-223, Jamie Shiers, CERN, 1211 Geneva 23, Switzerland

[6] Memorandum of Understanding for Collaboration in the Deployment and Exploitation of the Worldwide LHC Computing Grid, available at http://lcg.web.cern.ch/LCG/C-RRB/MoU/WLCGMoU.pdf.

[7] LCG Technical Design Report, CERN-LHCC-2005-024, available at http://lcg.web.cern.ch/LCG/tdr/.

[8] I. Foster, Argonne National Laboratory and University of Chicago, What is the Grid? A Three Point Checklist, 2002.

[9] Advances in Monitoring of Grid Services in WLCG, James Casey, Ian Neilson, to appear in the proceedings of the International Conference on Computing in High Energy Physics, Victoria, BC, September 2007.

[10] EGEE Grid Operations (SA1) - Site Monitoring and Visualization, presented at the EGEE'07 conference, October 2007, Budapest.

[11] EGEE Grid Operations (SA1 - JRA3) - Summary of progress with improving logging, presented at the EGEE'07 conference, October 2007, Budapest.

[12] LHC Computing Resources Review Board: http://lcg.web.cern.ch/LCG/Boards/crrb.html.

[13] Gridification: Porting New Communities onto the WLCG/EGEE Infrastructure, Patricia Méndez Lorenzo, Massimo Lamanna, Jakub Moscicki, Adrian Muraru EGEE User Forum, Manchester 10th May 2007.

[14] ITU, International Telecommunication Union Regional Radio Conference and the EGEE Grid, EGEE User Forum, CERN, 1st March 2006. Session: "Earth Observation-Archaeology—Digital library", Author: A. Manara, M. Cosic, T. Gavrilov, P.N. Hai.

[15] UNOSAT, Project Gridification: The UNOSAT Experience, EGEE User Forum, CERN, 1st March 2006. Session: "Earth ObservationArchaeology_Digital library", Author: P. Mendez.

[16] EGEE II news bulletins - http://www.eu-egee.org/news-listing.

[17] SETI@HOME - see http://setiweb.ssl.berkeley.edu/.

[18] LHC@HOME - see http://lhcathome.cern.ch/lhcathome/.

[19] The European Grid Initiative Design Study - website at http://www.euegi.org/. 